\title{
Various Models Mimicking the SM Higgs Boson a
}

\author{
Jung Chang ${ }^{1}$, Kingman Cheung ${ }^{1,2}$, Po-Yan Tseng ${ }^{1}$, and Tzu-Chiang Yuan ${ }^{3}$ \\ 1 Department of Physics, National Tsing Hua University, Hsinchu 300, Taiwan \\ 2 Division of Quantum Phases and Devices, School of Physics, \\ Konkuk University, Seoul 143-701, Republic of Korea \\ 3 Institute of Physics, Academia Sinica, Nangang, Taipei 11529, Taiwan
}

(Dated: October 22, 2018)

\begin{abstract}
This review is based on the talk presented at the SUSY 2012 (Beijing). The new particle around $125 \mathrm{GeV}$ observed at the Large Hadron Collider (LHC) is almost consistent with the standard model Higgs boson, except that the diphoton decay mode may be excessive. We summarize a number of possibilities. While at the LHC the dominant production mechanism for the Higgs boson of the standard model and some other extensions is via the gluon fusion process, the alternative vector-boson fusion is more sensitive to electroweak symmetry breaking. Using the well known dijet-tagging technique to single out the vector-boson fusion mechanism, we investigate potential of vector-boson fusion to discriminate a number of models suggested to give an enhanced inclusive diphoton production rate.
\end{abstract}

\footnotetext{
${ }^{a}$ Invited review in Int. J. Mod. Phys. A27, 1230030 (2012), based on the plenary talk given at the 20th International Conference on Supersymmetry and Unification of Fundamental Interactions (SUSY 2012), Peking University, Beijing, China August 13, 2012 - August 18, 2012
} 


\section{INTRODUCTION}

In this talk, we are going to summarize a few models that have been suggested to explain the newly observed particle of about $125 \mathrm{GeV}$ at the Large Hadron Collider (LHC) [1, 2]. It is of very high expectation that the observed particle is the long-sought Higgs boson, which was proposed in 1960s [3].

Before the LHC era there have been many speculations of the breaking of electroweak symmetry (EWSB). There are two known scales in particle physics - the electroweak scale and the Planck scale. The fundamental Higgs boson of order $100 \mathrm{GeV}$ is unstable against the radiative corrections. The so-called gauge hierarchy problem requires new physics has to come in around $\mathrm{TeV}$ scale in order that the unnatural cancellation between the bare mass term and the higher-order terms of the Higgs boson mass is under control. Historically, there are two categories of models: one with a strongly-coupled EWSB sector and one with a weakly-coupled EWSB sector. The most studied model for strong EWSB is the technicolortype model [4] while that for weakly-coupled EWSB model is the supersymmetry [5]. In technicolor models, the standard model (SM) is simply an effective model below TeV scale, at which the theory is replaced by another strong dynamics. Therefore, the cutoff scale now becomes just TeV. On the other hand, supersymmetric models predict another set of particles, which differ from their SM counter parts by a half-integral spin. Quadratically divergent contributions to the Higgs boson radiative corrections are cancelled among the SM particles and the corresponding supersymmetric particles.

If the Superconducting Super Collider (SSC) were built the Higgs boson could have been discovered in early 2000s with perhaps a short running of the machine. Since the Higgs boson has been hiding for such a long time, many interesting alternatives were proposed in the last 10-15 years. Around the turn of the century extra dimension models became very popular, not to mention there have been a large number of varieties- large extra dimension [6], universal extra dimension [7], Randall-Sundrum models [8], etc. There are also the

little Higgs type models [9]. In contrast to supersymmetry the new particles have the spin as their SM counter parts. Contributions to the Higgs boson radiative corrections are cancelled among the SM particles and the corresponding new particles. Perhaps, more and more models would have been proposed if the Higgs boson kept hiding. Wouldn't it be more fascinating for theorists? 
The past few years the high energy community has been very excited with a number of experimental anomalies from the Tevatron, LHC, and dark matter (DM) experiments. The long-time inconsistency among the DAMA results [10] (also the CoGeNT [11]) and the other direct detection experiments has motivated a number of unconventional dark matter models [12, 13], such as inelastic DM [14, isospin-violating DM [15], multi-component DM [16], etc. The top-quark forward-backward asymmetry observed by the CDF and D $\varnothing$ collaborations is rather puzzling too [17]. Many models such as flavor-changing $Z^{\prime}$, unusual $W^{\prime}$, axigluon, etc [18] were proposed, but the new LHC results seem ruled out almost all of these models [19]. The CDF $W j j$ anomaly in 2011 [20] also stimulated a large number of theoretical or phenomenological models to account for the observation [21]. However, with non-observation of the resonance from DØ and CMS 22] the excitement gradually died out.

At the end of 2011, both the ATLAS and CMS 23 experiments at the LHC have seen some excess of events of a possible Higgs candidate in the decays of $h \rightarrow \gamma \gamma, h \rightarrow W W^{*} \rightarrow$ $\ell \nu \ell \nu$ and $h \rightarrow Z Z^{*} \rightarrow 4 \ell$ channels. Finally, the discovery was jointly announced in July 2012 by ATLAS [1] and CMS [2]. All the observed channels, $W W, Z Z$ and $\gamma \gamma$ are consistent with the predictions of the SM Higgs boson, except that the $\gamma \gamma$ rate is somewhat higher than expectation. The $b \bar{b}$ and $\tau \tau$ channels are not confirmed yet, because of large uncertainties.

The diphoton production rate is about a factor of $1.3-2$ higher than that of the standard model Higgs boson, while the $Z Z^{*}$ and $W W^{*}$ rates are consistent with the SM Higgs boson within uncertainties. Nevertheless, the observed rates are consistent with either the SM Higgs boson or some other Higgs models. A large number of models have been put forward to account for the observed particle at $125 \mathrm{GeV}$, including the SM, MSSM, NMSSM, UMSSM and other MSSM-extended models, fermiophobic Higgs, 2HDM, RS radion, inertHiggs doublet, triplet Higgs models, etc.

The $H \rightarrow \gamma \gamma$ events collected by CMS and ATLAS can be divided into two categories: inclusive $\gamma \gamma X$ and exclusive $\gamma \gamma j j$ (though both experiments have more refined sub-divisions among various classes of events). Presumably, the inclusive $\gamma \gamma X$ events include all production channels such as gluon fusion, vector-boson fusion, associated production, etc, among which gluon fusion dominates for production of the SM Higgs boson and most of the models considered in this talk, except for the fermiophobic Higgs boson. On the other hand, exclusive $\gamma \gamma j j$ events mainly come from vector-boson fusion and associated production, which can be further disentangled by jet-tagging techniques. The vector-boson fusion produces ener- 
getic forward jets while associated production with a $W$ or $Z$ produces jets with $m_{j j} \approx m_{W}$ to $m_{Z}$. The current evidence of the Higgs boson in the diphoton channel comes mainly from inclusive $\gamma \gamma X$ events, simply because the inclusive event rate is much higher than the exclusive $\gamma \gamma j j$ event rate.

The main goal of this talk is to summarize all the models and find the parameter space of each model that have been proposed to explain the excess in the inclusive Higgs diphoton events, and attempt in distinguishing the models using the exclusive $\gamma \gamma j j$ channel in vectorboson fusion (VBF) [24]. The exclusive VBF events with $\gamma \gamma j j$ in the final state are selected using the forward jet-tagging techniques which will be explained shortly. We will first choose the parameter-space region of each model that can account for the excess in the inclusive diphoton rate, and then in that region of parameter space we calculate the exclusive $j j \gamma \gamma$ VBF production rates. We found that the exclusive $\gamma \gamma j j$ production rate in VBF channel can give more information to help in distinguishing a number of popular models.

We summarize a number of models that have been used to account for the excess in the inclusive $\gamma \gamma X$ data as follows.

1. The SM Higgs boson [3] is still believed to be the most desirable candidate. It is still consistent with the data within uncertainty.

2. The lighter Higgs boson of the minimal supersymmetric standard model (MSSM) can acquire a large radiative correction from the top-stop sector to achieve a mass of 125 $\mathrm{GeV}$, though it has been shown rather difficult to achieve an enhanced diphoton rate [25, 26]. However, it is possible when one of the staus is light enough, just above the LEP limit, and so the diphoton branching ratio is enhanced [25].

3. One of CP-even Higgs bosons in the next-to-minimal supersymmetric standard model (NMSSM) can account for the observed $125 \mathrm{GeV}$ boson with an enhanced diphoton rate [27]. It could be the lightest or the second lightest one. The $U(1)$-extended MSSM (UMSSM) [28] and other extensions [29] are also possible to account for the observed boson. The analyses for these extended MSSM models are much more involved and deserve dedicated studies.

4. The lighter CP-even Higgs boson of various types of the two-Higgs-doublet models (2HDM) [30], which has enough free parameters in the model that allows one to 
achieve a large branching ratio into $\gamma \gamma$.

5. In the fermiophobic (FP) Higgs boson model, the Higgs boson is only responsible to generate the masses to $W$ and $Z$ bosons while the fermion masses are generated by some other means. Since the FP Higgs boson does not couple to the quarks, it cannot be produced via gluon fusion at hadronic colliders, but only through the VBF and the associated production with a vector-boson. Nevertheless, the FP Higgs boson lighter than $130 \mathrm{GeV}$ has a much larger branching ratio into diphoton, such that it can still account for the observed inclusive diphoton rate at the LHC [31].

6. In Ref. [32], it was pointed out that the Randall-Sundrum (RS) radion, with enhanced couplings to $g g$ and $\gamma \gamma$ due to trace anomaly, can explain the excess in the inclusive diphoton production rate and suppressed $W W$ and $Z Z$ rates, which provides the most economical alternative solution to explain the observed data.

7. The inert-Higgs-doublet model (IHDM) [33], which is a special case of 2HDM, in which one of the doublets entirely decouples from the leptons, quarks, and gauge bosons while the other one takes on the role of the SM Higgs doublet. The production rate of the Higgs boson is the same as the SM one. However, the decay width of $h \rightarrow \gamma \gamma$ can be enhanced by the presence of the charged Higgs boson in the loop. It was shown 33 that the diphoton production rate can be enhanced by a factor of about $1-2$.

8. There may also be some possibilities that the SM-like Higgs boson first decays into two light scalar or pseudoscalar bosons, followed by subsequent decays into collimated pairs of photons, which appear as two photons in the final state [34]. On the other hand, instead of top-down approaches, it would also be useful to reversely determine the couplings and the nature of the observed $125 \mathrm{GeV}$ particle by studying all the available data [35].

The disadvantage of gluon fusion is that it is not clear what particles and their masses running in the triangle loop. In some models, the contribution from a particular charged particle can increase or decrease the diphoton decay width, depending on the relative signs. For example, in the supersymmetric models, there are additional sfermions, charginos, charged Higgs bosons running in the loop, and therefore resulting in complicated dependence on the model parameters. On the other hand, the advantage of using $W W$ fusion or associated 
production with a $W$ or a $Z$ boson is that the production diagram is clean and directly testing the couplings of $h W W$ and $h Z Z$. Furthermore, the $W W$ fusion has a cross section at least a factor of 2 larger than the associated production. We therefore focus on $W W$ fusion. The $W W$ fusion can be extracted by the presence of two energetic forward jets. We can impose selection cuts to select jets in forward rapidity and high energy region [36, 37]. By combining the production rates in the inclusive $\gamma \gamma X$ and exclusive $\gamma \gamma j j$ channels, one can obtain useful information about the nature of the $125 \mathrm{GeV}$ new particle recently observed at the LHC.

We calculate the event rates in the $W W$ fusion channel for a number of models that have been used to interpret the current LHC data of the $125 \mathrm{GeV}$ "Higgs boson". The theoretical cleanliness of $W W$ fusion has been explained in the last paragraph. We believe that the $W W$ fusion channel can provide useful information to discriminate various models. The organization of this paper is as follows. We briefly highlight a number of models in the next section, and the $W W$ fusion and selection cuts in Sec. III. We give the decay branching ratios in Sec. IV and production rates in Sec. V. We conclude in Sec. VI.

\section{MODELS}

\section{A. Standard Model Higgs Boson}

The SM Higgs boson [3] is still the most favorable candidate to interpret the observed boson, though the experimental data showed slightly excess in inclusive $\gamma \gamma X$ events over the prediction of the SM Higgs boson [1, 2]. Production of the SM Higgs boson is dominated by gluon fusion, which is an order of magnitude larger than the next important mechanism $-\mathrm{VBF}$.

\section{B. Two-Higgs-Doublet Model (2HDM)}

There are two Higgs doublets instead of just one in the 2HDM. In order to avoid dangerous tree level flavor-changing neutral currents, the popular 2HDMs are imposed a discrete symmetry. In the type I, all of the fermions couple to a single Higgs doublet, and do not

couple to the second doublet; while in the type II, one doublet couples only to down-type quarks and another doublet couples to the up-type quarks. In this talk, we focus on the 
type II, which has the same Higgs sector as the MSSM. The Higgs sector consists of two Higgs doublets

$$
H_{u}=\left(\begin{array}{c}
H_{u}^{+} \\
H_{u}^{0}
\end{array}\right), \quad H_{d}=\left(\begin{array}{c}
H_{d}^{+} \\
H_{d}^{0}
\end{array}\right),
$$

where the subscripts $u, d$ denote the right-handed quark singlet field that the Higgs doublet couples to. The electroweak symmetry is broken when the Higgs doublet fields develop the following VEVs:

$$
\left\langle H_{u}\right\rangle=\left(\begin{array}{c}
0 \\
v_{u}
\end{array}\right), \quad\left\langle H_{d}\right\rangle=\left(\begin{array}{c}
0 \\
v_{d}
\end{array}\right) .
$$

Physically, there are two CP-even, one CP-odd, and a pair of charged Higgs bosons after electroweak symmetry breaking (EWSB), and the $W$ and $Z$ bosons as well as the SM fermions, except for neutrinos, acquire masses. The Yukawa couplings and masses for fermions can be obtained from the following Yukawa interactions after EWSB

$$
\mathcal{L}_{\text {Yuk }}=-y_{u} \overline{Q_{L}} u_{R} \tilde{H}_{u}-y_{d} \overline{Q_{L}} d_{R} H_{d}+\text { h.c. }
$$

where $\tilde{H}_{u}=i \tau_{2} H_{u}^{*}$. The parameters of the model in the CP-conserving case include

$$
m_{h}, m_{H}, m_{A}, m_{H^{+}}, \tan \beta \equiv \frac{v_{u}}{v_{d}}, \alpha
$$

where $\alpha$ is the mixing angle between the two CP-even Higgs bosons. There are enough free parameters in the Higgs potential such that all the above parameters are free inputs to the model, in contrast to the MSSM where the Higgs potential is highly restricted by supersymmetry in addition to gauge symmetry.

The couplings of the two lighter and heavier CP-even Higgs bosons $h$ and $H$ respectively and the CP-odd Higgs boson $A$ to the top, bottom quarks, and taus are given by, with a common factor of $-i g m_{f} / 2 m_{W}$ being suppressed,

$$
\begin{array}{cccc} 
& t \bar{t} & b \bar{b} & \tau^{-} \tau^{+} \\
h: & \cos \alpha / \sin \beta & -\sin \alpha / \cos \beta & -\sin \alpha / \cos \beta \\
H: & \sin \alpha / \sin \beta & \cos \alpha / \cos \beta & \cos \alpha / \cos \beta \\
A: & -i \cot \beta \gamma_{5} & -i \tan \beta \gamma_{5} & -i \tan \beta \gamma_{5}
\end{array}
$$

while the charged Higgs $H^{-}$couples to $t$ and $\bar{b}$ via

$$
\bar{b} t H^{-}: \quad \frac{i g}{2 \sqrt{2} m_{W}}\left[m_{t} \cot \beta\left(1+\gamma_{5}\right)+m_{b} \tan \beta\left(1-\gamma_{5}\right)\right] .
$$


Other relevant couplings in $W W$ fusion are those to gauge bosons are given by,

$$
\begin{aligned}
h W^{+} W^{-}: & i g m_{W} \sin (\beta-\alpha) g^{\mu \nu} \\
h Z Z & : \quad i g m_{Z} \frac{\sin (\beta-\alpha)}{\cos \theta_{W}} g^{\mu \nu} .
\end{aligned}
$$

Dominant production of the light CP-even Higgs boson $h$ at the LHC is via gluon fusion, similar to the SM Higgs boson with the top quark running in the loop; however, in the large $\tan \beta$ region the bottom-quark contribution can also be substantial. Since the bottomYukawa coupling can be substantially enhanced, the gluon fusion cross section can be larger than the SM. On the other hand, since the couplings of the $h$ to the $W W$ and $Z Z$ are simply the SM values multiplied by $\sin (\alpha-\beta), W W$ fusion cross sections are in general smaller than the SM.

The decay into two photons is somewhat more complicated than the SM. Besides the couplings $h W W, h t \bar{t}$, and $h b \bar{b}$ are different, there are also the charged Higgs bosons running in the loop. The charged Higgs boson couples to the light CP-even Higgs with the coupling 26

$$
\lambda_{h H^{+} H^{-}}=\frac{m_{h}^{2}-\lambda_{5} v^{2}}{m_{W}^{2}} \cos (\beta+\alpha)+\frac{2 m_{H^{ \pm}}^{2}-m_{h}^{2}}{2 m_{W}^{2}} \sin (2 \beta) \sin (\beta-\alpha) .
$$

However, the $b \rightarrow s \gamma$ and $\mathrm{B}$ meson mixing constraints require the charged Higgs boson mass $m_{H^{ \pm}}>500 \mathrm{GeV}$ for intermediate to large values of $\tan \beta$ [38]. We will choose $m_{H^{ \pm}}=500$ $\mathrm{GeV}$ in our analysis below.

The overall diphoton production rate $\sigma(g g \rightarrow h) \times B(h \rightarrow \gamma \gamma)$ in gluon fusion can easily vary between $0.5-2$ of the SM prediction depending on parameters [30]. It was shown in Ref. [30] that the enhancement in branching ratio can be obtained roughly along sin $\alpha$ near zero for all $\tan \beta$ in the type II model. We choose parameter space points there to illustrate.

\section{Supersymmetric Higgs boson: MSSM}

In order to achieve a mass of $125 \mathrm{GeV}$ for the lighter CP-even Higgs boson, a very large

radiative correction is needed, which essentially comes from top-stop loop. The approximate formula for the lighter CP-even Higgs boson is given by [25]

$$
m_{h}^{2} \approx m_{Z}^{2} \cos ^{2} 2 \beta+\frac{3 m_{t}^{4}}{4 \pi^{2} v^{2}}\left[\frac{1}{2} X_{t}+t+\frac{1}{16 \pi^{2}}\left(\frac{3 m_{t}^{2}}{2 v^{2}}-32 \pi \alpha_{s}\right) t\left(X_{t}+t\right)\right]
$$


where

$$
X_{t}=\frac{2\left(A_{t}-\mu \cot \beta\right)^{2}}{M_{\mathrm{SUSY}}^{2}}\left(1-\frac{\left(A_{t}-\mu \cot \beta\right)^{2}}{12 M_{\mathrm{SUSY}}^{2}}\right), \quad t=\frac{M_{\mathrm{SUSY}}^{2}}{m_{t}^{2}}
$$

and $M_{\mathrm{SUSY}} \sim 1 \mathrm{TeV}$ is the SUSY scale. A large $A_{t}$ is needed to generate a large correction. Here we follow the findings in Ref. [25] for the parameter space: we choose $m_{Q_{3}}=m_{U_{3}}=850$ $\mathrm{GeV}, A_{t}=1.4 \mathrm{TeV}, m_{A}=1 \mathrm{TeV}$, and $\tan \beta=60$. A detailed analysis of the MSSM parameter space based on Bayesian statistical analysis in light of the new observation of the $125 \mathrm{GeV}$ Higgs candidate is also presented recently in Ref. [39]. The reason behind such a large $\tan \beta$ is the stau contribution to the diphoton branching ratio explained below [25].

In the production part via gluon fusion, the difference between the SM and supersymmetric models is that squarks also run in the triangle loop. As the experimental data have pushed the squark masses of the first two generations to be quite heavy but not the third generation (stop and sbottom) the change in production rates could be substantial, especially in large $\tan \beta$. On the other hand, the decay into diphoton is more involved in SUSY models. All charged particles, including squarks, sleptons, charginos, charged Higgs boson can flow in the triangle loop. With the present constraints from experiments, the production rate into diphoton (equal to production cross section times the branching ratio into diphoton) in the MSSM is shown to be very similar to the SM one and that the diphoton production rate can hardly be enhanced by more than a factor of 1.5 [25, 26, 39].

The formulas for the decay of the Higgs boson into two photons as well as production via gluon fusion can be found in Ref. [40]. The couplings of the lighter CP-even Higgs boson to the $W W$ or $Z Z$ are given by the SM ones multiplied by $\sin (\alpha-\beta)$. Therefore, the production rate in the $W W$ fusion is in general similar to or smaller than the SM prediction.

We look at the parameter space in which the diphoton production rate would be larger than the SM value in the MSSM. It was shown in Ref. [25] that diphoton production rate can be larger than the SM one if one pushes the stau to be very light, just above the LEP limit. In addition to the above mentioned soft parameters, the other parameters are $m_{L_{3}}$, $m_{E_{3}}$, and the $\mu$. Without loss of generality we choose [25]

$$
m_{L_{3}}=m_{e_{3}}=200-450 \mathrm{GeV} \quad \text { and } \quad \mu=200-1000 \mathrm{GeV}
$$

in which we can scan for the diphoton production rate $\sigma(g g \rightarrow h) B(h \rightarrow \gamma \gamma)$ to be larger than the SM rate. The region essentially gives a light stau, which can enhance the $B(h \rightarrow$ 
$\gamma \gamma$ ). We will scan the region according to Eq. (4) and require the mass of the lighter CP-even Higgs boson around $125 \mathrm{GeV}$ and the diphoton production rate larger than the SM value. 1

\section{Fermiophobic Higgs}

With the name "fermiophobic" (FP) the Higgs boson only couples to the vector bosons at tree level, though higher-loop corrections can induce small couplings to fermions. In this case, the Yukawa couplings and masses of fermions are generated by some other mechanisms, which are not of concern in this talk.

The coupling strength of the FP Higgs boson to vector bosons is the same as that of the SM Higgs boson. We write the interactions as

$$
\mathcal{L}_{\mathrm{FP}}=-g m_{W} h_{\mathrm{FP}} W_{\mu}^{+} W^{-\mu}-\frac{g m_{Z}}{2 \cos \theta_{W}} h_{\mathrm{FP}} Z_{\mu} Z^{\mu} .
$$

Since the FP Higgs boson does not couple to quarks, it cannot be produced dominantly by gluon fusion at hadronic colliders, but only through the vector-boson fusion and the associated production with a $W / Z$ boson. The corresponding production cross sections are the same as the VBF of the SM Higgs boson. Nevertheless, the FP Higgs boson lighter than $130 \mathrm{GeV}$ has a much larger branching ratio into diphoton, such that it can still account for the observed diphoton rate at the LHC [31]. There are two reasons: (i) the FP Higgs boson decay into fermions is highly suppressed with only the loop-induced couplings, and (ii) the decay into photons is via a loop of $W$ boson without the negative interference from the top quark. Thus, the branching ratio into diphoton can be enhanced by more than an order of magnitude. Overall, the diphoton production rate at the LHC is comparable to the SM Higgs boson, as was used to account for the observed boson [31]. An earlier study of FP Higgs boson at the LHC can be found in Ref. [42]. There is basically no free parameters in this model.

\section{E. The Radion}

The RS model [8] used a warped 5D space-time, a slice of the symmetric space $A d S_{5}$, to explain the gauge hierarchy problem. The extra dimension $\varphi$ is a single $S^{1} / Z_{2}$ orbifold with

${ }^{1}$ There is another possibility that the heavier CP-even Higgs boson can be at around $125 \mathrm{GeV}$ and its diphoton production rates can be enhanced relative to the SM one [41]. We will not pursue this further here. 
one hidden and one visible 3-brane localized at $\varphi=0$ and $\pi$, respectively. It was pointed out by Goldberger and Wise [43] that the original RS model has a four-dimensional massless scalar (the modulus or radion) which does not have a potential and therefore the extra dimension cannot be stabilized. A stabilization mechanism was proposed in [43] by adding a bulk scalar field propagating in the background solution which can generate a potential to stabilize the modulus field. The minimum of the potential can be arranged to give the desired value of $k r_{c} \sim 12$ to solve the gauge hierarchy problem without extreme fine tuning of parameters. As a consequence, the lightest excitation mode of the modulus field is the radion, which has a mass of the order of $100 \mathrm{GeV}$ to a $\mathrm{TeV}$, and the strength of its coupling to the SM fields is of the order of $O(1 / \mathrm{TeV})$ [44]. Phenomenology of the stabilized radion and its effects on the background geometry were studied in [45].

The interactions of the stabilized modulus (radion) $\phi$ with the SM particles on the visible brane are completely determined by 4 -dimensional general covariance. Thus the radion Lagrangian is given by

$$
\mathcal{L}_{\text {radion }}=\frac{\phi}{\Lambda_{\phi}} T_{\mu}^{\mu}(\mathrm{SM}),
$$

where $\Lambda_{\phi}=\langle\phi\rangle$ is of the order of $\mathrm{TeV}$ and $T_{\mu}^{\mu}$ is the trace of the SM energy-momentum stress tensor, which has the following lowest order terms

$$
T_{\mu}^{\mu}(\mathrm{SM})=-2 m_{W}^{2} W_{\mu}^{+} W^{-\mu}-m_{Z}^{2} Z_{\mu} Z^{\mu}+\sum_{f} m_{f} \bar{f} f+\left(2 m_{h}^{2} h^{2}-\partial_{\mu} h \partial^{\mu} h\right)+\cdots
$$

The coupling of the radion to a pair of gluons (photons) is induced at one loop level, with the dominated contributions coming from the heavy top quark (top quark and $W$ ) as well as from the trace anomaly in QCD (QED). The expressions of the induced couplings can be found in Ref.[32]. Similar to the SM Higgs boson, the dominant production channel for the radion is via $g g$ fusion, followed by VBF [46]. In addition, $g g \rightarrow \phi$ gets substantial enhancement from the trace anomaly. For the decay of the radion, it is dominated by the $g g$ mode instead of $b \bar{b}$ at the low mass region, while its diphoton branching ratio is merely a fraction of the SM value of $h_{\mathrm{SM}} \rightarrow \gamma \gamma$.

Overall, the diphoton production rate $\sigma(g g \rightarrow \phi) \times B(\phi \rightarrow \gamma \gamma)$ can be larger than the $\mathrm{SM}$ rate if the scale $\Lambda_{\phi}$ is small enough, and as long as it is consistent with the search for RS graviton. If we do not concern about naturalness, the scale $\Lambda_{\phi}$ can be as small as $0.8 \mathrm{TeV}$. Here we fix the scale $\Lambda_{\phi}$ to be $0.8-0.99 \mathrm{TeV}$, which can enhance the diphoton production 
rate in gluon fusion by a factor of $1.5-1.0$ [32] relative to the SM rate. Note that the branching ratios of the radion is independent of $\Lambda_{\phi}$.

\section{F. Inert Higgs Doublet Model (IHDM)}

IHDM is a special case of $2 \mathrm{HDM}$, in which one of the doublets takes on the role of the SM Higgs doublet, while the other one is inert which means that it entirely decouples from the SM leptons, quarks, and gauge bosons. The model also has an additional $Z_{2}$ symmetry, for which all SM particles are even, except for the particle content of the second inert Higgs doublet. The lightest $Z_{2}$-odd particle of the second doublet can work as a candidate of dark matter. The Higgs sector consists of

$$
H_{1}=\left(\begin{array}{c}
\phi_{1}^{+} \\
\frac{v}{\sqrt{2}}+\frac{h+i \chi}{\sqrt{2}}
\end{array}\right), \quad H_{2}=\left(\begin{array}{c}
\phi_{2}^{+} \\
\frac{S+i A}{\sqrt{2}}
\end{array}\right) .
$$

The electroweak symmetry is broken solely by one VEV:

$$
\left\langle H_{1}\right\rangle=\left(\begin{array}{c}
0 \\
\frac{v}{\sqrt{2}}
\end{array}\right), \quad\left\langle H_{2}\right\rangle=\left(\begin{array}{l}
0 \\
0
\end{array}\right) .
$$

The Higgs potential is given by

$V=\mu_{1}^{2}\left|H_{1}\right|^{2}+\mu_{2}^{2}\left|H_{2}\right|^{2}+\lambda_{1}\left|H_{1}\right|^{4}+\lambda_{2}\left|H_{2}\right|^{4}+\lambda_{3}\left|H_{1}\right|^{2}\left|H_{2}\right|^{2}+\lambda_{4}\left|H_{1}^{\dagger} H_{2}\right|^{2}+\frac{\lambda_{5}}{2}\left[\left(H_{1}^{\dagger} H_{2}\right)^{2}+\right.$ h.c. $]$

Physically, there are 2 CP-even scalars $(h, S), 1$ CP-odd scalar $(A)$, and a pair of charged Higgs $\left(H^{ \pm}\right)$. The $h$ plays the role of the SM Higgs boson while the others are inert. A list of parameters of the model includes $m_{h}, m_{S}, m_{A}, m_{H^{ \pm}}, \mu_{2}$, and $\lambda_{2}$.

Production via gluon fusion and via $W W$ fusion are the same as the SM Higgs boson. However, the decay into $\gamma \gamma$ receives additional contributions from the $H^{ \pm}$running in the loop. If kinematically allowed the Higgs boson $h$ can also decay into $H^{+} H^{-}, A A$, and $S S$. For simplicity and to achieve a large enough branching ratio into $\gamma \gamma$ we set the masses of $S, A, H^{+}$to be above the threshold $\left(m_{h} / 2\right)$. In this model, the coupling between the charged Higgs boson and the SM Higgs boson $h$ is given by

$$
g_{h H^{+} H^{-}}=-i \frac{e}{m_{W} \sin \theta_{W}}\left(m_{H^{ \pm}}^{2}-\mu_{2}^{2}\right) .
$$

It is clear that from this equation the sign of the charged Higgs contribution to the triangular loop can be positive or negative, depending on the sizes of $m_{H^{+}}$and $\mu_{2}$. Thus, if we set 


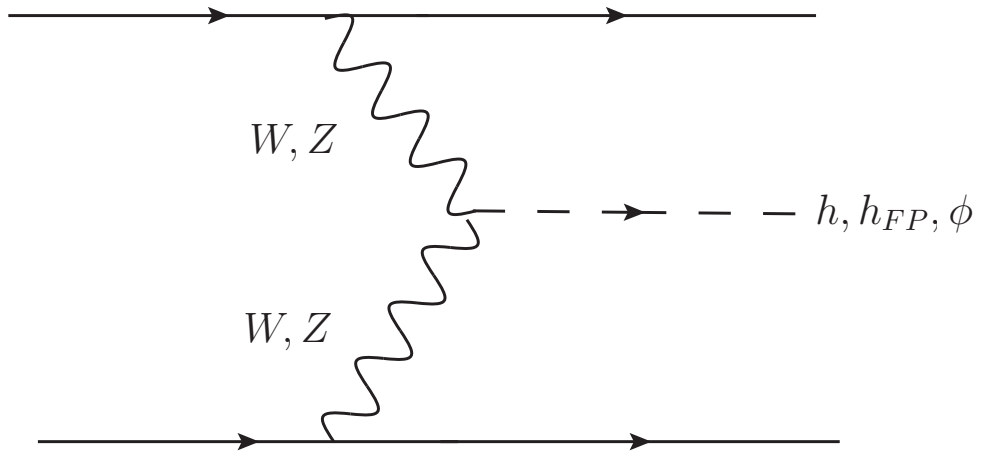

FIG. 1. A Feynman diagram showing the vector-boson fusion into SM Higgs, FP Higgs or radion.

$m_{H^{+}}=\mu_{2}$, the charged Higgs contribution vanishes and so the diphoton branching ratio becomes the same as the SM one.

It was shown in Ref. [33] that in gluon fusion the diphoton production rate is determined by the product of gluon-fusion cross section and the $\gamma \gamma$ branching ratio

$$
\frac{\sigma(g g \rightarrow h) B(h \rightarrow \gamma \gamma)}{\sigma(g g \rightarrow h)_{\mathrm{SM}} B(h \rightarrow \gamma \gamma)_{\mathrm{SM}}}=\frac{B(h \rightarrow \gamma \gamma)}{B(h \rightarrow \gamma \gamma)_{\mathrm{SM}}}
$$

which can be varied from about 0.5 to 2. The charged Higgs contribution to the diphoton branching ratio depends on the sizes of $m_{H^{ \pm}}$and $\mu_{2}$. It was shown in Ref. [33] that when $m_{H^{ \pm}}^{2}<\mu_{2}^{2}$ the diphoton branching ratio is enhanced. Together with other theoretical constraints one can find the region to be $\left|\mu_{2}\right| \approx 100-200 \mathrm{GeV}$ and $m_{H^{ \pm}}<\left|\mu_{2}\right|$.

\section{VECTOR-BOSON FUSION (VBF)}

The most distinguished feature of $\mathrm{VBF}$ at hadronic colliders is the appearance of two energetic forward jets separated by a large $\Delta R=\sqrt{(\Delta \eta)^{2}+(\Delta \phi)^{2}}$, where $\eta$ is the pseudorapidity and $\phi$ is the azimuth angle. The Feynman diagram is shown in Fig. 1. Each of the initial quarks radiates a $W / Z$ boson, which further annihilates into the Higgs boson or some other particles under consideration. The unique feature of this process is that the $W / Z$ bosons participating in the fusion process are close to on-shell [47], and irrespecive whether the $W / Z$ is longitudinal or transverse, the scattered quark carries almost all the energy of the incoming quark and goes in the forward direction." [36, 37]. This fact justifies the use of effective $W$ approximation in all calculations for VBF in the early days. Based on this feature we impose the following experimental cuts in selecting the dijet events coming 
dominantly from the VBF:

$$
E_{T_{j}}>30 \mathrm{GeV},\left|\eta_{j}\right|<4.7, \Delta R_{j j}>3.5
$$

and

$$
\begin{array}{ll}
\text { (Ejcut) } & E_{j_{1}}>500 \mathrm{GeV} \quad \text { or } \\
\text { (Mjjcut) } & M_{j j}>350 \mathrm{GeV},
\end{array}
$$

where the subscript "1" denotes the most energetic jet. In the cut of Eq. (11), we choose either the energy of the most energetic jet $E_{j_{1}}>500 \mathrm{GeV}$ or the invariant mass of the jet pair $M_{j j}>350 \mathrm{GeV}$. This set of cuts is similar to that used by CMS [48] and ATLAS [49] in their searches for FP Higgs boson.

The vector-boson fusion is well-known that it allows to probe the direct coupling between the vector bosons and the Higgs boson or other particles under consideration. This is in contrast to the $g g$ fusion, because any colored particles can flow in the triangular loop and affect the production rate. For example, in MSSM all squarks can circulate inside the loop.

\section{DECAY BRANCHING RATIOS}

Here we list the decay branching ratio into $\gamma \gamma$ for the SM Higgs boson, fermiophobic Higgs boson, and the radion of mass $125 \mathrm{GeV}$ in Table I. For the SM Higgs boson the decay into $\gamma \gamma$ goes through a triangle loop of $W$ boson and top quark, between which they interfere destructively. The SM Higgs diphoton branching ratio is about $2.3 \times 10^{-3}$. This is very different for the FP Higgs boson, which allows only the $W$ boson flowing in the loop. Thus, the branching ratio of a FP Higgs boson into diphoton can be an order of magnitude larger than that of the SM Higgs boson, and also because it does not decay into fermions. That is the reason why it can account for the observed 125 Higgs boson at the LHC, even though its gluon fusion production cross section is very small. The case of the radion is opposite to the FP Higgs. The diphoton branching ratio of the radion is a few times smaller than that of the SM, while its production rate via $g g$ fusion is substantially enhanced.

We also list the branching ratio into $\gamma \gamma$ for the Higgs boson in the IHDM for a number of choices of parameters of the model in Table I], such that the diphoton branching ratio is enhanced relative to the SM one: $\left|\mu_{2}\right| \approx 100-200 \mathrm{GeV}$ and $m_{H^{ \pm}}<\left|\mu_{2}\right|$. The factors affecting 
TABLE I. Decay branching ratio $B(h \rightarrow \gamma \gamma)$ for the SM Higgs boson $h_{\mathrm{SM}}$, the fermiophobic Higgs boson $h_{F P}$, the radion $\phi$, the inert Higgs doublet model, the two Higgs doublet model, and the MSSM.

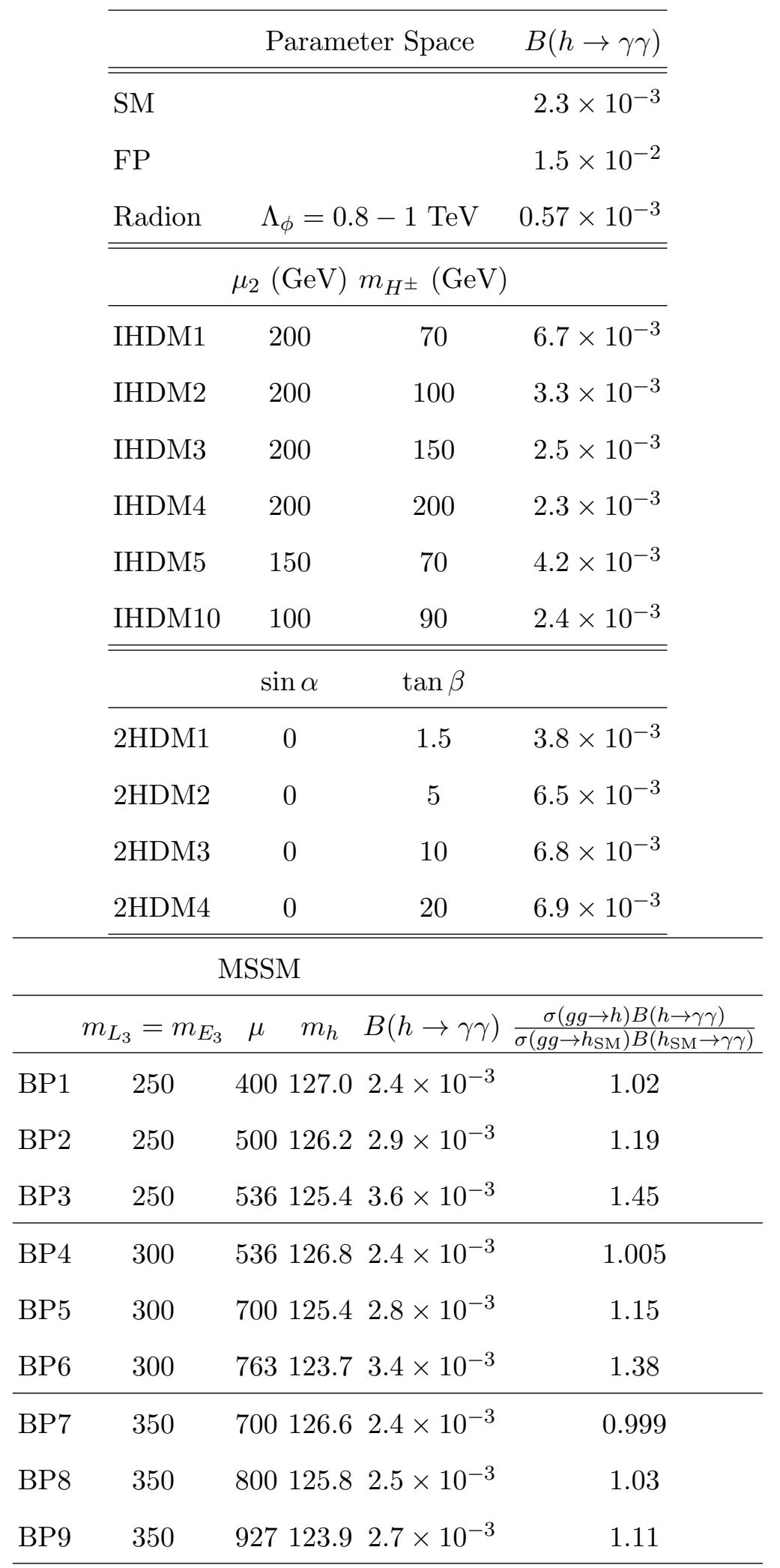


the partial width into $\gamma \gamma$ are the charged Higgs boson mass and the coupling $g_{h H^{+} H^{-}}$. The charged Higgs loop contribution can interfere either constructively or destructively with the SM contributions. Another factor that would affect the branching ratio into $\gamma \gamma$ is whether the thresholds into $S S, A A$, or $H^{+} H^{-}$are open. However, for our choices for $m_{S}, m_{H^{+}}$and $m_{A}$ these decays would not be allowed. The diphoton branching ratio can be made similar to the SM one or enhanced by a few times.

The branching ratios into $\gamma \gamma$ for the light CP-even Higgs boson in the 2HDM for a number of choices of parameters of the model are shown in Table I, such that the enhancement in branching ratio can be achieved roughly along $\sin \alpha \approx 0$. Similar to IHDM, the main factors affecting the partial width into $\gamma \gamma$ are the charged Higgs boson mass and the coupling $g_{h H^{+} H^{-}}$. The branching ratio, on the other hand, also depends on other parameters such as $\tan \beta$ and $\sin \alpha$ as exhibited in the $h W W$ and $h t \bar{t}$ couplings. Along $\sin \alpha \approx 0$, the factor $\cos \alpha \approx 1$ and the factor $\sin (\beta-\alpha) \approx 1$ for large $\tan \beta$, and thus the couplings $h W W$ and $h t \bar{t}$ are close to their SM values. On the other hand, along $\sin \alpha=-1$ for large $\tan \beta$, the $h W W$ coupling proportional to $\sin (\beta-\alpha)$ is only about $1 / \tan \beta$. That is the reason why its branching ratio into $\gamma \gamma$ is very small.

In the MSSM, we choose the region where the lighter CP-even Higgs boson is around $125 \mathrm{GeV}(123-128 \mathrm{GeV})$ and the diphoton production rate $\sigma(g g \rightarrow h) B(h \rightarrow \gamma \gamma)$ is equal to or larger than the SM value. As explained above the stop sector must be heavy in order to achieve a mass of $125 \mathrm{GeV}$ for the lighter CP-even Higgs boson. First, we fix the $m_{Q_{3}}=m_{U_{3}}=850 \mathrm{GeV}$ and $A_{t}=1.4 \mathrm{TeV}$ with $\tan \beta=60$ and $m_{A}=1 \mathrm{TeV}$. Second, we vary $m_{E_{3}}=m_{L_{3}}$ and $\mu$ to achieve a light stau so as to enhance the diphoton production rate, according to Eq. (4). We used the FeynHiggs [50] to evaluate the branching ratio $B(h \rightarrow \gamma \gamma)$ and the diphoton production rate $\sigma(g g \rightarrow h) B(h \rightarrow \gamma \gamma)$ relative to the $\mathrm{SM}$ values. We show in Fig. 2 the region in the plane of $\left(m_{E_{3}}=m_{L_{3}}, \mu\right)$ that the $m_{h}=123-128 \mathrm{GeV}$ and the ratio $\sigma(g g \rightarrow h) B(h \rightarrow \gamma \gamma) / \sigma\left(g g \rightarrow h_{\mathrm{SM}}\right) B\left(h_{\mathrm{SM}} \rightarrow \gamma \gamma\right)$ is larger than 1 . We also show the branching ratios into $\gamma \gamma$ for the lighter CP-even Higgs boson and the inclusive diphoton production rate for a few selective points of MSSM in Table 1 . 


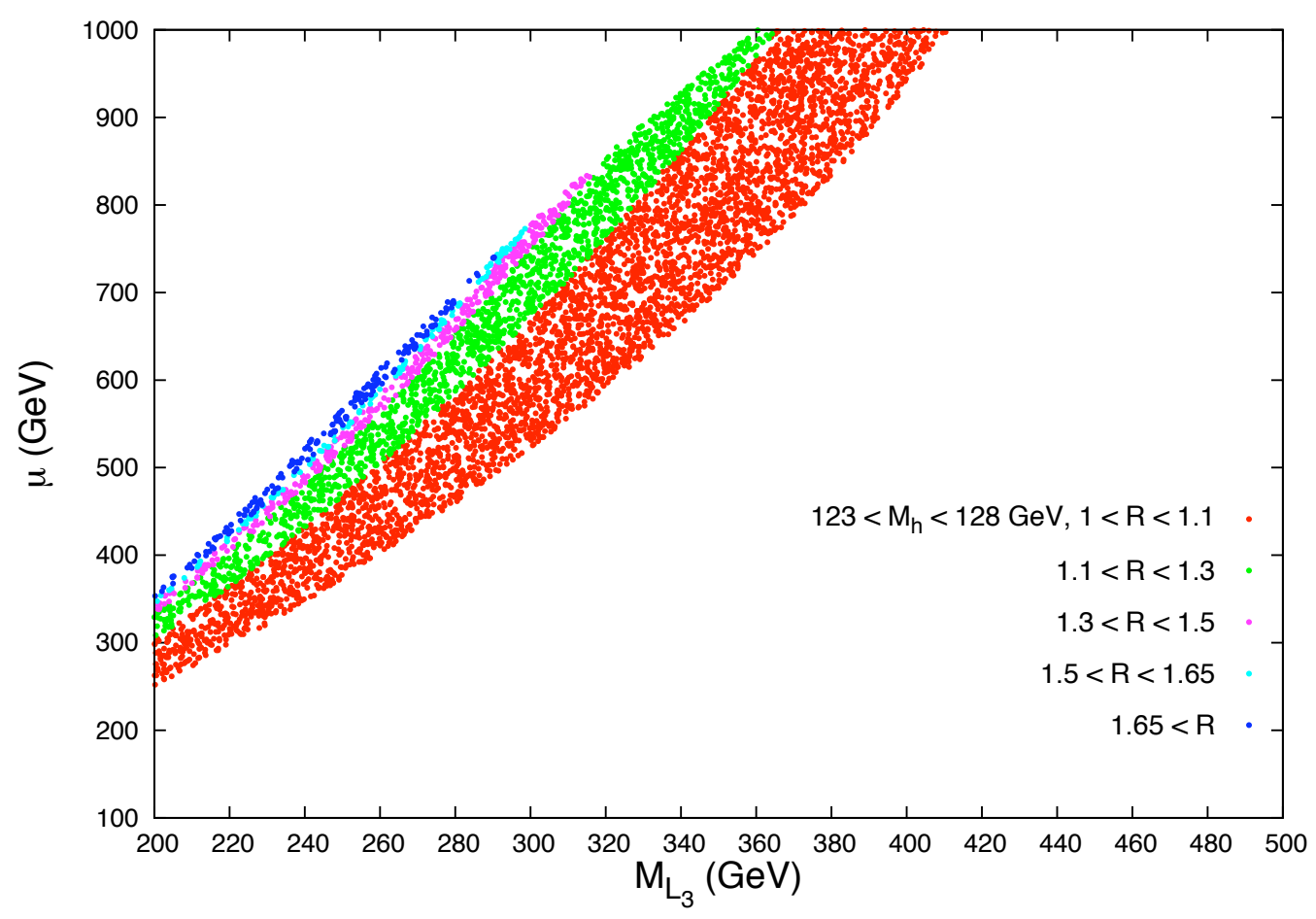

FIG. 2. The parameter space region where $m_{h}=123-128 \mathrm{GeV}$ and $R=\sigma(g g \rightarrow h) B(h \rightarrow$ $\gamma \gamma) / \sigma\left(g g \rightarrow h_{\mathrm{SM}}\right) B\left(h_{\mathrm{SM}} \rightarrow \gamma \gamma\right)$ is larger than 1 . The other fixed parameters are $m_{Q_{3}}=m_{U_{3}}=850$ $\mathrm{GeV}, A_{t}=1.4 \mathrm{TeV}, \tan \beta=60$ and $m_{A}=1 \mathrm{TeV}$.

\section{PRODUCTION RATES IN VECTOR-BOSON FUSION}

We calculate the VBF cross sections for $p p \rightarrow j j h$ for various models under consideration. We impose the selection cuts for energetic forward jets as in Eqs. (10) and (11). We let the Higgs boson decay into $\gamma \gamma$, and impose the following cuts on the photons:

$$
E_{T_{\gamma}}>30 \mathrm{GeV}, \quad\left|\eta_{\gamma}\right|<2.5, \quad\left|m_{\gamma \gamma}-m_{h}\right|<3.5 \mathrm{GeV} .
$$

In the following, we present numerical results in both parton level and detector-simulation level by employing the PYTHIA-PGS (PYTHIA v6.420, PGS4(090401)) package inside MADGRAPH [51].

We employ MADGRAPH [51] to calculate the production cross sections for $p p \rightarrow j j h \rightarrow$ $j j \gamma \gamma$ and implementing the selection cuts for the forward jets and the diphoton. The produc- 
TABLE II. Production rates in fb at PGS level for $p p \rightarrow j j h \rightarrow j j \gamma \gamma$ at LHC-7 (LHC-8, LHC-14)

\begin{tabular}{|c|c|c|}
\hline & \multicolumn{2}{|c|}{ photon cuts and Ejcut photon cuts and Mjjcut } \\
\hline $\mathrm{SM}$ & $0.15(0.19,0.61)$ & $0.33(0.41,1.1)$ \\
\hline $\mathrm{FP}$ & $1.03(1.27,4.12)$ & $2.24(2.78,7.35)$ \\
\hline Radion & $0.0038(0.0047,0.014)$ & $0.0076(0.0095,0.026)$ \\
\hline IDHM1 & $0.44(0.56,1.79)$ & $0.97(1.21,3.23)$ \\
\hline IDHM2 & $0.22(0.28,0.88)$ & $0.48(0.59,1.59)$ \\
\hline IDHM3 & $0.16(0.21,0.67)$ & $0.36(0.45,1.21)$ \\
\hline IDHM4 & $0.15(0.19,0.62)$ & $0.33(0.41,1.11)$ \\
\hline IDHM5 & $0.28(0.35,1.12)$ & $0.61(0.76,2.03)$ \\
\hline IDHM10 & $0.16(0.20,0.64)$ & $0.35(0.43,1.16)$ \\
\hline 2HDM1 & $0.17(0.22,0.70)$ & $0.38(0.47,1.25)$ \\
\hline 2HDM2 & $0.41(0.52,1.66)$ & $0.90(1.11,2.99)$ \\
\hline 2HDM3 & $0.44(0.56,1.79)$ & $0.97(1.20,3.23)$ \\
\hline 2HDM4 & $0.45(0.58,1.85)$ & $1.00(1.24,3.33)$ \\
\hline \multicolumn{3}{|c|}{ MSSM BP photon cuts and Ejcut photon cuts and Mjjcut } \\
\hline BP1 & $0.19(0.28,0.83)$ & $0.44(0.57,1.47)$ \\
\hline BP2 & $0.22(0.33,0.97)$ & $0.52(0.66,1.76)$ \\
\hline BP3 & $0.29(0.40,1.18)$ & $0.63(0.82,2.10)$ \\
\hline BP4 & $0.19(0.28,0.85)$ & $0.43(0.56,1.46)$ \\
\hline BP5 & $0.22(0.32,0.92)$ & $0.50(0.65,1.65)$ \\
\hline BP6 & $0.27(0.38,1.07)$ & $0.61(0.75,1.90)$ \\
\hline BP7 & $0.20(0.26,0.85)$ & $0.43(0.53,1.47)$ \\
\hline BP8 & $0.21(0.29,0.85)$ & $0.44(0.59,1.48)$ \\
\hline BP9 & $0.22(0.30,0.92)$ & $0.49(0.59,1.66)$ \\
\hline
\end{tabular}

tion rates of diphoton for various models are then obtained by multiplying the corresponding diphoton branching ratios.

We use PYTHIA [52] for parton showering and hadronization. During the parton showering we turn on the initial and final state QED and QCD radiations (ISR and FSR), and fragmentation/hadronization according to the Lund model. We use PGS [53] for detector- 
simulation with the most general settings for the LHC. Electromagnetic and hadronic calorimeter resolutions are set at $0.2 / \sqrt{E}$ and $0.8 / \sqrt{E}$, respectively. We use the cone algorithm for jet finding with a cone size of $\Delta R=0.5$, Sagitta resolution of $13 \mu \mathrm{m}$ $\left(\delta p_{T} / p_{T}=1.04 \times 10^{-4}\right)$, track-finding efficiency of 0.98 , and minimum track $p_{T}$ of $1 \mathrm{GeV}$. More details can be found in Refs. [51, 53.

The numerical results for LHC-7, LHC-8, and LHC-14 are listed in Table II for the SM, FP, radion, IHDM, 2HDM, and the MSSM.

It would be more transparent to show the production rate relative to the SM one

$$
\frac{\sigma(p p \rightarrow j j X) \times B(X \rightarrow \gamma \gamma)}{\sigma\left(p p \rightarrow j j h_{\mathrm{SM}}\right) \times B\left(h_{\mathrm{SM}} \rightarrow \gamma \gamma\right)}
$$

where $X$ stands for the SM Higgs or any other Higgs-like candidate in various models. We found that this ratio is quite robust against various cuts (Mjjcut or Ejcut as in Eq. (11)) and against the energy of the collision.

In the upper panel of Fig. 3, we first show the ratio for the inclusive diphoton production rate $\frac{\sigma(X) \times B(X \rightarrow \gamma \gamma)}{\sigma\left(h_{\mathrm{SM}}\right) \times B\left(h_{\mathrm{SM}} \rightarrow \gamma \gamma\right)}$ of each model, which is dominated by gluon fusion, except for the FP Higgs boson. The parameter space of each model is chosen such that this inclusive diphoton rate is equal to or larger than the SM rate except for the FP Higgs boson which has no free parameter. For the same parameter space, we show in the lower panel the ratio for the exclusive $j j \gamma \gamma$ production rate $\frac{\sigma(p p \rightarrow j j X) \times B(X \rightarrow \gamma \gamma)}{\sigma\left(p p \rightarrow j j h_{\mathrm{SM}}\right) \times B\left(h_{\mathrm{SM}} \rightarrow \gamma \gamma\right)}$ for each model. The figure is valid for LHC-7, LHC-8 and LHC-14, and for using either Mjjcut cut on both forward jets or Ejcut cut on the most energetic jet.

It is clear that the models can be employed to explain the excess in the inclusive diphoton rates in some parameter space region, but for the same region of parameter space the ratio of exclusive VBF production would be different among the models. The FP Higgs would be a number of times larger than the SM in the VBF channel, but the RS radion would give negligible exclusive VBF production. On the other hand, the IHDM, 2HDM, and the MSSM would give similar ratios in both inclusive and exclusive production. The 2HDM can give a somewhat smaller ratio in the exclusive VBF. 

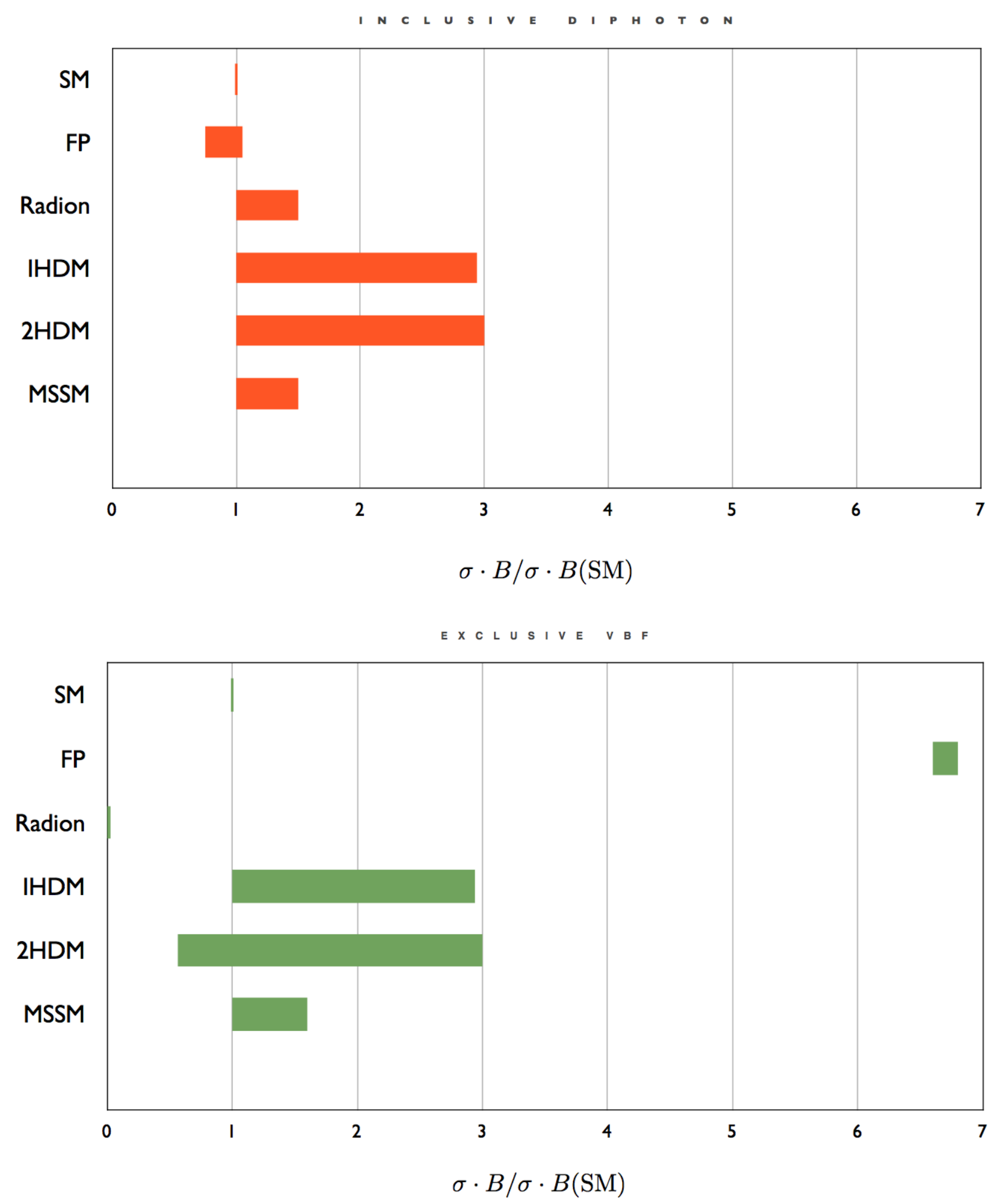

FIG. 3. Upper: the ratio of inclusive diphoton production rates $\frac{\sigma(X) \times B(X \rightarrow \gamma \gamma)}{\sigma\left(h_{\mathrm{SM}}\right) \times B\left(h_{\mathrm{SM}} \rightarrow \gamma \gamma\right)}$ for various models. The parameter space is chosen such that the ratio is equal to or larger than 1, except for the FP Higgs boson which has no free parameter. Lower: the ratio of the exclusive $j j \gamma \gamma$ production rate $\frac{\sigma(p p \rightarrow j j X) \times B(X \rightarrow \gamma \gamma)}{\sigma\left(p p \rightarrow j j h_{\mathrm{SM}}\right) \times B\left(h_{\mathrm{SM}} \rightarrow \gamma \gamma\right)}$ for each model in the corresponding parameter space. 


\section{A. Background Discussion}

The experimental details for VBF in the context of searching for the FP Higgs boson are given in Ref. [48]. The dominant backgrounds to $h \rightarrow \gamma \gamma$ consist of i) the irreducible background from the prompt diphoton production, and ii) the reducible backgrounds from $p p \rightarrow \gamma+j$ and $p p \rightarrow j j$, when one or more of the jets hadronize into (typically neutral) pions and deposit energy in the electromagnetic calorimeter. These reconstructed objects are generally referred to as fake photons. Isolation is a very useful tool to reject the nonprompt background coming from electromagnetic showers originating in jets, because these fake photons are often accompanied by single and multiple $\pi^{0} \mathrm{~s}$.

The dijet tag, as defined in Eqs. (10) and (11), together with the Higgs boson decaying into diphoton selects a special class of events consisting of two photons and two forward energetic jets. A signal-to-background ratio of order 1 can be achieved [48]. The signal from $h \rightarrow \gamma \gamma$ will be identified as a sharp peak in the $m_{\gamma \gamma}$ distribution, where the background is in continuum. In Ref. [48, the background model is derived from data. In the new run

at $8 \mathrm{TeV}$, we expect the same treatment is applied to the background. The estimation of significance of each signal is beyond the scope of the present paper.

Given that we have obtained the event rates of each model in the tables, which have been under experimental cuts and simulations (PGS), it is straight-forward to estimate the required luminosity to probe various scenarios. The continuum background at the $7 \mathrm{TeV}$ and $8 \mathrm{TeV}$ LHC in VBF channel has been obtained in Refs. [48] and [2]. Since the SM Higgs boson has been seen above the background with some significance level in the VBF channel in the current LHC runs (about $5 \mathrm{fb}^{-1}$ at $7 \mathrm{TeV}$ and about $5 \mathrm{fb}^{-1}$ at $8 \mathrm{TeV}$ ), the scenarios with higher event rates than the SM Higgs boson should be detectable. The projected integrated luminosity at the end of 2012 is about $10-15 \mathrm{fb}^{-1}$ for each experiment, it would not be a problem to investigate the scenarios in the VBF channel.

\section{CONCLUSIONS}

LHC is expected to confirm if there is a new particle at $125 \mathrm{GeV}$ by the end of this year. The likelihood for a new discovery is rather high. Nevertheless, whether this new particle is the SM Higgs boson is not an easy question to answer. 
Here the scenario of this $125 \mathrm{GeV}$ particle produced by vector-boson fusion, instead of gluon fusion as the dominant production mechanism for the standard model Higgs boson, is studied in details. By using the forward dijet tagging technique, one can single out the vector-boson fusion mechanism. We studied a number of popular new physics models that have been employed to interpret the observed particle at $125 \mathrm{GeV}$, including fermiophobic Higgs boson, the Randall-Sundrum radion, inert-Higgs-doublet model, two-Higgs-doublet model, and the MSSM. Since the inclusive diphoton channel showed an excess over the SM predictions, we first selected the parameter space of each model that can give an inclusive diphoton rate larger than or equal to the SM rate. Then, we calculate the exclusive $j j \gamma \gamma$ diphoton production rate in VBF for that parameter space. If the diphoton mode excess seen at LHC-7 can be firmly established by the new LHC-8 data, it will be the utmost task to identify the nature of this particle. Perhaps, it is simply the SM Higgs boson with some level of statistical fluctuation, but it could also be the RS radion [32], fermiophobic Higgs boson [31], the light CP-even Higgs boson of the 2HDM [30], the Higgs boson of the IHDM [33], or one of the CP-even Higgs bosons in other extensions of the MSSM [27, 28], all of which can allow an enhancement to the $\gamma \gamma$ production rate. On the other hand, the vector-boson fusion, as singled out by the dijet tag, provides useful information in helping to differentiate among various models. It is not hard by browsing through Fig. 3 to conclude the following

- If a similar rate is seen in inclusive production but no large excess is seen in the exclusive VBF production it would unlikely be a fermiophobic Higgs boson.

- If a similar rate or excess is seen in inclusive production but some events are seen in exclusive VBF production rate, it would unlikely be the RS radion.

- If moderate excess is seen in both inclusive production and exclusive VBF production, it could be the Higgs boson of the IHDM, 2HDM, or the MSSM. However, if the excess is over $60 \%$ it will pose severe challenge to the MSSM.

It seems easy to rule out either fermiophobic Higgs boson or RS radion, providing that we see no large excess or some events in VBF channel, respectively. However, it is still difficult to distinguish the other models when the inclusive and exclusive rates are similar to or slightly larger than the SM values. If the production rate of the diphoton mode at $125 \mathrm{GeV}$ lines up 
with the SM prediction eventually, it is still premature to conclude this is coming from the SM Higgs boson. Other alternatives in MSSM, NMSSM, or other SUSY models can also be mimicking the SM Higgs boson, depending on the parameter space of the new physics model. In any case, once the signals at $125 \mathrm{GeV}$ are confirmed further studies including all possible decay modes are to be taken into account in order to discriminate these many alternatives beyond the standard model.

Vector-boson fusion is the next most important production mechanism that must be taken into account to fully identify the newly discovered particle.

\section{ACKNOWLEDGMENT}

This work was supported in part by the National Science Council of Taiwan under Grants No. 99-2112-M-007-005-MY3 and No. 101-2112-M-001-005-MY3 as well as the WCU program through the KOSEF funded by the MEST (R31-2008-000-10057-0).

[1] G. Aad et al. [The ATLAS Collaboration], arXiv:1207.7214 [hep-ex].

[2] S. Chatrchyan et al. [The CMS Collaboration], arXiv:1207.7235 [hep-ex].

[3] P. W. Higgs, Phys. Rev. Lett. 13, 508 (1964); F. Englert and R. Brout, Phys. Rev. Lett. 13, 321 (1964); G. S. Guralnik, C. R. Hagen and T. W. B. Kibble, Phys. Rev. Lett. 13, 585 (1964).

[4] E. Farhi and L. Susskind, Phys. Rept. 74, 277 (1981).

[5] H. E. Haber and G. L. Kane, Phys. Rept. 117, 75 (1985).

[6] N. Arkani-Hamed, S. Dimopoulos and G. R. Dvali, Phys. Rev. D 59, 086004 (1999) [hep$\mathrm{ph} / 9807344]$.

[7] T. Appelquist, H. -C. Cheng and B. A. Dobrescu, Phys. Rev. D 64, 035002 (2001) [hep$\mathrm{ph} / 0012100]$.

[8] L. Randall and R. Sundrum, Phys. Rev. Lett. 83, 3370 (1999) [hep-ph/9905221].

[9] N. Arkani-Hamed, A. G. Cohen, E. Katz and A. E. Nelson, JHEP 0207, 034 (2002) [hep$\mathrm{ph} / 0206021]$. 
[10] R. Bernabei et al. [DAMA and LIBRA Collaborations], Eur. Phys. J. C 67, 39 (2010) [arXiv:1002.1028 [astro-ph.GA]].

[11] C. E. Aalseth et al. [CoGeNT Collaboration], Phys. Rev. Lett. 106, 131301 (2011) [arXiv:1002.4703 [astro-ph.CO]].

[12] Z. Ahmed et al. [CDMS-II Collaboration], Science 327, 1619 (2010) [arXiv:0912.3592 [astroph.CO]].

[13] E. Aprile et al. [XENON100 Collaboration], arXiv:1207.5988 [astro-ph.CO].

[14] D. Tucker-Smith and N. Weiner, Phys. Rev. D 64, 043502 (2001) [hep-ph/0101138].

[15] J. L. Feng, J. Kumar, D. Marfatia and D. Sanford, Phys. Lett. B 703, 124 (2011) [arXiv:1102.4331 [hep-ph]].

[16] K. M. Zurek, Phys. Rev. D 79, 115002 (2009) [arXiv:0811.4429 [hep-ph]].

[17] T. Aaltonen et al. [CDF Collaboration], Phys. Rev. Lett. 101, 202001 (2008) [arXiv:0806.2472 [hep-ex]]; T. Aaltonen et al. [CDF Collaboration], Phys. Rev. D 83, 112003 (2011) [arXiv:1101.0034 [hep-ex]]; V. M. Abazov et al. [D0 Collaboration], Phys. Rev. D 84, 112005 (2011) [arXiv:1107.4995 [hep-ex]].

[18] S. Jung, H. Murayama, A. Pierce and J. D. Wells, Phys. Rev. D 81, 015004 (2010) [arXiv:0907.4112 [hep-ph]]; K. Cheung, W. -Y. Keung and T. -C. Yuan, Phys. Lett. B 682, 287 (2009) [arXiv:0908.2589 [hep-ph]]; J. Shu, T. M. P. Tait and K. Wang, Phys. Rev. D 81, 034012 (2010) [arXiv:0911.3237 [hep-ph]]; P. H. Frampton, J. Shu and K. Wang, Phys. Lett. B 683, 294 (2010) [arXiv:0911.2955 [hep-ph]].

[19] S. Chatrchyan et al. [CMS Collaboration], Phys. Lett. B 709, 28 (2012) [arXiv:1112.5100 [hepex]]; J. A. Aguilar-Saavedra and M. Perez-Victoria, JHEP 1109, 097 (2011) [arXiv:1107.0841 [hep-ph]].

[20] T. Aaltonen et al. [CDF Collaboration], Phys. Rev. Lett. 106, 171801 (2011) [arXiv:1104.0699 [hep-ex]].

[21] K. Cheung and J. Song, Phys. Rev. Lett. 106, 211803 (2011) [arXiv:1104.1375 [hep-ph]]; A. E. Nelson, T. Okui and T. S. Roy, Phys. Rev. D 84, 094007 (2011) [arXiv:1104.2030 [hepph]]. X. -P. Wang, Y. -K. Wang, B. Xiao, J. Xu and S. -h. Zhu, Phys. Rev. D 83, 115010 (2011) [arXiv:1104.1917 [hep-ph]]. F. Yu, Phys. Rev. D 83, 094028 (2011) [arXiv:1104.0243 [hep-ph]]; M. R. Buckley, D. Hooper, J. Kopp and E. Neil, Phys. Rev. D 83, 115013 (2011) [arXiv:1103.6035 [hep-ph]]. P. J. Fox, J. Liu, D. Tucker-Smith and N. Weiner, Phys. Rev. D 
84, 115006 (2011) [arXiv:1104.4127 [hep-ph]]; E. J. Eichten, K. Lane and A. Martin, Phys. Rev. Lett. 106, 251803 (2011) [arXiv:1104.0976 [hep-ph]].

[22] V. M. Abazov et al. [D0 Collaboration], Phys. Rev. Lett. 107, 011804 (2011) [arXiv:1106.1921 [hep-ex]]; S. Chatrchyan et al. [CMS Collaboration], arXiv:1208.3477 [hep-ex].

[23] G. Aad et al. [ATLAS Collaboration], Phys. Lett. B 710, 49 (2012) [arXiv:1202.1408 [hepex]]; S. Chatrchyan et al. [CMS Collaboration], Phys. Lett. B 710, 26 (2012) [arXiv:1202.1488 [hep-ex]].

[24] J. Chang, K. Cheung, P. -Y. Tseng and T. -C. Yuan, arXiv:1206.5853 [hep-ph].

[25] M. Carena, S. Gori, N. R. Shah and C. E. M. Wagner, JHEP 1203, 014 (2012) [arXiv:1112.3336 [hep-ph]]; M. Carena, S. Gori, N. R. Shah, C. E. M. Wagner and L. -T. Wang, JHEP 1207, 175 (2012) [arXiv:1205.5842 [hep-ph]].

[26] H. Baer, V. Barger and A. Mustafayev, Phys. Rev. D 85, 075010 (2012) [arXiv:1112.3017 [hepph]]; S. Heinemeyer, O. Stal and G. Weiglein, Phys. Lett. B 710, 201 (2012) [arXiv:1112.3026 [hep-ph]]; A. Arbey, M. Battaglia, A. Djouadi, F. Mahmoudi and J. Quevillon, Phys. Lett. B 708, 162 (2012) [arXiv:1112.3028 [hep-ph]]; P. Draper, P. Meade, M. Reece and D. Shih, Phys. Rev. D 85, 095007 (2012) [arXiv:1112.3068 [hep-ph]]; S. Akula, B. Altunkaynak, D. Feldman, P. Nath and G. Peim, Phys. Rev. D 85, 075001 (2012) [arXiv:1112.3645 [hep-ph]]; M. Kadastik, K. Kannike, A. Racioppi and M. Raidal, JHEP 1205, 061 (2012) [arXiv:1112.3647 [hep-ph]]; J. Cao, Z. Heng, D. Li and J. M. Yang, Phys. Lett. B 710, 665 (2012) [arXiv:1112.4391 [hep-ph]]; N. D. Christensen, T. Han and S. Su, arXiv:1203.3207 [hep-ph]; J. L. Feng and D. Sanford, Phys. Rev. D 86, 055015 (2012) [arXiv:1205.2372 [hep-ph]]; J. -J. Cao, Z. X. Heng, J. M. Yang, Y. -M. Zhang and J. -Y. Zhu, JHEP 1203, 086 (2012) [arXiv:1202.5821 [hep-ph]]; T. Li, J. A. Maxin, D. V. Nanopoulos and J. W. Walker, Phys. Lett. B 710, 207 (2012) [arXiv:1112.3024 [hep-ph]]; V. Barger, M. Ishida and W. -Y. Keung, arXiv:1207.0779 [hep-ph].

[27] U. Ellwanger, JHEP 1203, 044 (2012) [arXiv:1112.3548 [hep-ph]]; J. F. Gunion, Y. Jiang and S. Kraml, Phys. Lett. B 710, 454 (2012) [arXiv:1201.0982 [hep-ph]]; S. F. King, M. Muhlleitner and R. Nevzorov, Nucl. Phys. B 860, 207 (2012) [arXiv:1201.2671 [hep-ph]]; J. Cao, Z. Heng, J. M. Yang, Y. Zhang and J. Zhu, JHEP 1203, 086 (2012) [arXiv:1202.5821 [hep-ph]]; U. Ellwanger and C. Hugonie, arXiv:1203.5048 [hep-ph]; D. A. Vasquez, G. Belanger, C. Boehm, J. Da Silva, P. Richardson and C. Wymant, Phys. Rev. D 86, 035023 (2012) [arXiv:1203.3446 
[hep-ph]].

[28] C. -F. Chang, K. Cheung, Y. -C. Lin and T. -C. Yuan, JHEP 1206128 (2012), [arXiv:1202.0054 [hep-ph]].

[29] M. Hirsch, W. Porod, L. Reichert and F. Staub, arXiv:1206.3516 [hep-ph]; H. An, T. Liu and L. -T. Wang, arXiv:1207.2473 [hep-ph]; P. Athron, S. F. King, D. J. Miller, S. Moretti and R. Nevzorov, arXiv:1206.5028 [hep-ph]; K. S. Jeong, Y. Shoji and M. Yamaguchi, JHEP 1209, 007 (2012) [arXiv:1205.2486 [hep-ph]]; F. Boudjema and G. D. La Rochelle, Phys. Rev. D 86, 015018 (2012) [arXiv:1203.3141 [hep-ph]]; P. Fileviez Perez, Phys. Lett. B 711, 353 (2012) [arXiv:1201.1501 [hep-ph]].

[30] P. M. Ferreira, R. Santos, M. Sher and J. P. Silva, Phys. Rev. D 85, 077703 (2012) [arXiv:1112.3277 [hep-ph]]; G. Burdman, C. E. F. Haluch and R. D. Matheus, Phys. Rev. D 85, 095016 (2012) [arXiv:1112.3961 [hep-ph]]; E. Cervero and J. -M. Gerard, Phys. Lett. B 712, 255 (2012) [arXiv:1202.1973 [hep-ph]]; P. M. Ferreira, R. Santos, M. Sher and J. P. Silva, Phys. Rev. D 85, 035020 (2012) [arXiv:1201.0019 [hep-ph]]; N. Chen and H. -J. He, JHEP 1204, 062 (2012) [arXiv:1202.3072 [hep-ph]]; A. Arhrib, R. Benbrik and C. -H. Chen, arXiv:1205.5536 [hep-ph]; H. S. Cheon and S. K. Kang, arXiv:1207.1083 [hep-ph]; W. Altmannshofer, S. Gori and G. D. Kribs, arXiv:1210.2465 [hep-ph]; N. Craig and S. Thomas, arXiv:1207.4835 [hep-ph]; D. S. M. Alves, P. J. Fox and N. J. Weiner, arXiv:1207.5499 [hep-ph]; S. Chang, S. K. Kang, J. -P. Lee, K. Y. Lee, S. C. Park and J. Song, arXiv:1210.3439 [hep-ph]; Y. Bai, V. Barger, L. L. Everett and G. Shaughnessy, arXiv:1210.4922 [hep-ph]; A. Drozd, B. Grzadkowski, J. F. Gunion and Y. Jiang, arXiv:1211.3580 [hep-ph]; T. Abe, N. Chen and H. -J. He, arXiv:1207.4103 [hep-ph].

[31] E. Gabrielli, B. Mele and M. Raidal, arXiv:1202.1796 [hep-ph]; E. L. Berger, Z. Sullivan and H. Zhang, arXiv:1203.6645 [hep-ph]; E. Gabrielli, K. Kannike, B. Mele, A. Racioppi and M. Raidal, Phys. Rev. D 86, 055014 (2012) [arXiv:1204.0080 [hep-ph]].

[32] K. Cheung and T. -C. Yuan, Phys. Rev. Lett. 108, 141602 (2012) [arXiv:1112.4146 [hep-ph]]; V. Barger, M. Ishida and W. -Y. Keung, Phys. Rev. Lett. 108, 101802 (2012) [arXiv:1111.4473 [hep-ph]]; ibid. Phys. Rev. D 85, 015024 (2012) [arXiv:1111.2580 [hep-ph]]; B. Grzadkowski, J. F. Gunion and M. Toharia, Phys. Lett. B 712, 70 (2012) [arXiv:1202.5017 [hep-ph]]; Y. Tang, arXiv:1204.6145 [hep-ph]; S. Matsuzaki and K. Yamawaki, Phys. Rev. D 85, 095020 (2012) [arXiv:1201.4722 [hep-ph]]; H. de Sandes and R. Rosenfeld, Phys. Rev. D 85, 053003 
(2012) [arXiv:1111.2006 [hep-ph]].

[33] A. Arhrib, R. Benbrik and N. Gaur, Phys. Rev. D 85, 095021 (2012) [arXiv:1201.2644 [hepph]]; L. Wang and X. -F. Han, JHEP 1205, 088 (2012) [arXiv:1203.4477 [hep-ph]].

[34] P. Draper and D. McKeen, Phys. Rev. D 85, 115023 (2012) [arXiv:1204.1061 [hep-ph]].

[35] D. Carmi, A. Falkowski, E. Kuflik and T. Volansky, arXiv:1202.3144 [hep-ph]; A. Azatov, R. Contino and J. Galloway, JHEP 1204, 127 (2012) [arXiv:1202.3415 [hep-ph]]; J. R. Espinosa, C. Grojean, M. Muhlleitner and M. Trott, JHEP 1205, 097 (2012) [arXiv:1202.3697 [hep-ph]]; V. Barger, M. Ishida and W. -Y. Keung, Phys. Rev. Lett. 108, 261801 (2012) [arXiv:1203.3456 [hep-ph]]; P. P. Giardino, K. Kannike, M. Raidal and A. Strumia, JHEP 1206, 117 (2012) [arXiv:1203.4254 [hep-ph]]; J. Ellis and T. You, JHEP 1206, 140 (2012) [arXiv:1204.0464 [hep-ph]]; A. Azatov, R. Contino, D. Del Re, J. Galloway, M. Grassi and S. Rahatlou, JHEP 1206, 134 (2012) [arXiv:1204.4817 [hep-ph]]; M. Klute, R. Lafaye, T. Plehn, M. Rauch and D. Zerwas, arXiv:1205.2699 [hep-ph].

[36] J. Bagger, V. D. Barger, K. -m. Cheung, J. F. Gunion, T. Han, G. A. Ladinsky, R. Rosenfeld and C. -P. Yuan, Phys. Rev. D 52, 3878 (1995) [hep-ph/9504426].

[37] D. L. Rainwater and D. Zeppenfeld, JHEP 9712, 005 (1997) [hep-ph/9712271].

[38] K. Cheung and O. C. W. Kong, Phys. Rev. D 68, 053003 (2003) [hep-ph/0302111].

[39] S. Akula, P. Nath and G. Peim, arXiv:1207.1839 [hep-ph]; P. Nath, arXiv:1210.0520 [hep-ph].

[40] J. F. Gunion, H. E. Haber, G. L. Kane and S. Dawson, Front. Phys. 80, 1 (2000).

[41] K. Hagiwara, J. S. Lee and J. Nakamura, arXiv:1207.0802 [hep-ph].

[42] A. G. Akeroyd, J. Phys. G G 24, 1983 (1998) [hep-ph/9803324].

[43] W. Goldberger and M. Wise, Phys. Rev. Lett. 83, 4922 (1999).

[44] W. Goldberger and M. Wise, Phys. Lett. B475 275 (2000).

[45] C. Csáki, M. Graesser, L. Randall and J. Terning, Phys. Rev. D 62, 045015 (2000); C. Csáki, M. L. Graesser and G. D. Kribs, Phys. Rev. D 63, 065002 (2001) [hep-th/0008151].

[46] K. -m. Cheung, Phys. Rev. D 63, 056007 (2001) [hep-ph/0009232]; W. D. Goldberger, B. Grinstein and W. Skiba, Phys. Rev. Lett. 100, 111802 (2008) [arXiv:0708.1463 [hep-ph]].

[47] M. S. Chanowitz, Ann. Rev. Nucl. Part. Sci. 38, 323 (1988).

[48] CMS Collaboration, "Search for the fermiophobic model Higgs boson decaying into two photons", CMS-PAS-HIG-12-002, March 2012. 
[49] ATLAS Collaboration, "Search for a fermiophobic Higgs boson in the diphoton decay channel with $4.9 \mathrm{fb}^{1}$ of ATLAS data at $\sqrt{s}=7$ TeV", ATLAS-CONF-2012-13, March 2012.

[50] M. Frank, T. Hahn, S. Heinemeyer, W. Hollik, H. Rzehak and G. Weiglein, JHEP 0702, 047 (2007) [hep-ph/0611326].

[51] MADGRAPH: J. Alwall, M. Herquet, F. Maltoni, O. Mattelaer and T. Stelzer, JHEP 1106, 128 (2011) [arXiv:1106.0522 [hep-ph]].

[52] T. Sjostrand, S. Mrenna and P. Z. Skands, Comput. Phys. Commun. 178, 852 (2008) [arXiv:0710.3820 [hep-ph]].

[53] The MG/ME Pythia-PGS package http://madgraph.hep.uiuc.edu/index.html, J. Alwall and the CP3 development team. 